\title{
MEETING REPORT OPEN \\ Summary of workshop on sub-seasonal to seasonal predictability of extreme weather and climate
}

\author{
Andrew W. Robertson ${ }^{1}$, Suzana J. Camargo ${ }^{2}$, Adam Sobel ${ }^{2,4}$, Frederic Vitart ${ }^{3}$ and Shuguang Wang ${ }^{4}$
}

This paper provides a summary of the Workshop on Sub-Seasonal to Seasonal (S2S) Predictability of Extreme Weather and Climate, held at Columbia University, December 6-7, 2016. The 2-day workshop was attended by over 100 people and took stock of recent developments in Sub-seasonal to Seasonal predictability, S2S extreme weather phenomena, and real world predictions and use of forecasts. Workshop motivations, new findings, and outstanding questions discussed are described.

npj Climate and Atmospheric Science (2018)1:20178; doi:10.1038/s41612-017-0009-1

\section{INTRODUCTION}

Columbia University's International Research Institute for Climate and Society (IRI) and its Initiative on Extreme Weather and Climate, together with the World Weather Research Program (WWRP) and World Climate Research Program (WCRP)'s Sub-Seasonal to Seasonal Prediction Project (S2S), with support from NOAA's Modeling, Analysis, Predictions and Projections (MAPP) program and involvement of its S2S Prediction Task Force, held a 2-day workshop on December 6-7, 2016 at the Columbia University Lamont-Doherty Earth Observatory campus in Palisades, New York. The workshop targeted all in academia, government, and the private sector with an interest in understanding the latest science behind S2S predictability of extreme weather and climate and in developing early warning products. Some 115 people attended in person, with another 150 joining the online conference livestream. The workshop consisted of 23 talks, 33 posters, and discussion sessions, with the goal of sharing the latest research on extremes using S2S models, the WWRP/WCRP S2S research initiative and data archive, operational S2S forecasting, private sector forecasts, sources of S2S predictability of weather extremes, and risk management perspectives. These talks are briefly summarized below, and a list of the presenters and presentation titles is provided in Table 1. Several of the talks have been developed into papers appearing in this special issue. The workshop program can be accessed at http://iri.columbia.edu/s2s-extremes-workshop2016/ where recorded videos of the presentations can be accessed.

The workshop was motivated by the increasing interest in extreme weather and climate events, both in terms of developing the potential for early warning systems for better societal preparedness, as well as toward gaining a better understanding of the potential impacts of climate change on disruptive weather. Recent scientific developments ${ }^{1}$ in sub-seasonal to seasonal prediction (from 2 weeks to a season ahead), together with the establishment of the WWRP/WCRP S2S prediction project archive of operational model forecasts, provide a new opportunity to better understand the physical phenomena and processes that cause extreme weather and climate events with large societal impacts (floods, droughts, storms, heat, and cold waves), as well as to improve their prediction for early warning. Two weeks to a season ahead is a key forecasting time range, both from the physical perspective of the climatic drivers of extremes (MJO, blocking events, sudden stratospheric warmings, land/sea/ice surface interactions), and for decision makers to have sufficient time to take preemptive actions. However, it is also a challenging range because it falls into a gap that currently exists between weather and seasonal climate forecasts: weather is deterministically predictable as a function of atmospheric initial conditions up to a theoretical limit of a week or two, while climate acquires probabilistic predictability from slowly varying atmospheric boundary conditions (notably the ocean) on seasonal and longer scales. The $\sim 2$ weeks to a season range was previously thought to be a "predictability desert", where neither had much power. Recent research on sources of predictability and improved modeling has started to change that picture. The workshop summarized the key advances in this emerging area. From the user standpoint, sub-seasonal forecasts made on a near-weekly basis, could fill the gap between weather forecasts that are issued every day, and seasonal climate forecasts that are typically only made once a month; they have the potential to provide weekly updated forecast information of intermediate specificity about the risks of extremes up to several weeks ahead that may be particularly amenable to a range of users.

\section{SUB-SEASONAL TO SEASONAL PREDICTABILITY}

The workshop began with a series of talks on S2S predictability of large-scale atmospheric phenomena, beginning with an introduction to the S2S project, which is a 5-year joint research project of the WWRP and WCRP that began in 2013. ${ }^{2}$ A central pillar of the S2S project is a large research data archive of S2S forecasts and hindcasts from 11 operational forecasting centers around the world. $^{3}$ The talk was given by F. Vitart (ECMWF) who is one of the co-chairs of the S2S project, together with A. W. Robertson (IRI). The S2S project has already advanced research significantly

\footnotetext{
${ }^{1}$ International Research Institute for Climate and Society (IRI), Columbia University, Palisades, NY, USA; ${ }^{2}$ Lamont-Doherty Earth Observatory, Columbia University, Palisades, NY,

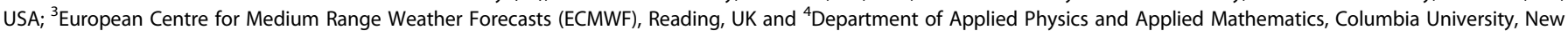
York, NY, USA

Correspondence: Andrew W. Robertson (awr@iri.columbia.edu)
}

Received: 31 May 2017 Revised: 1 September 2017 Accepted: 15 September 2017

Published online: 21 February 2018 


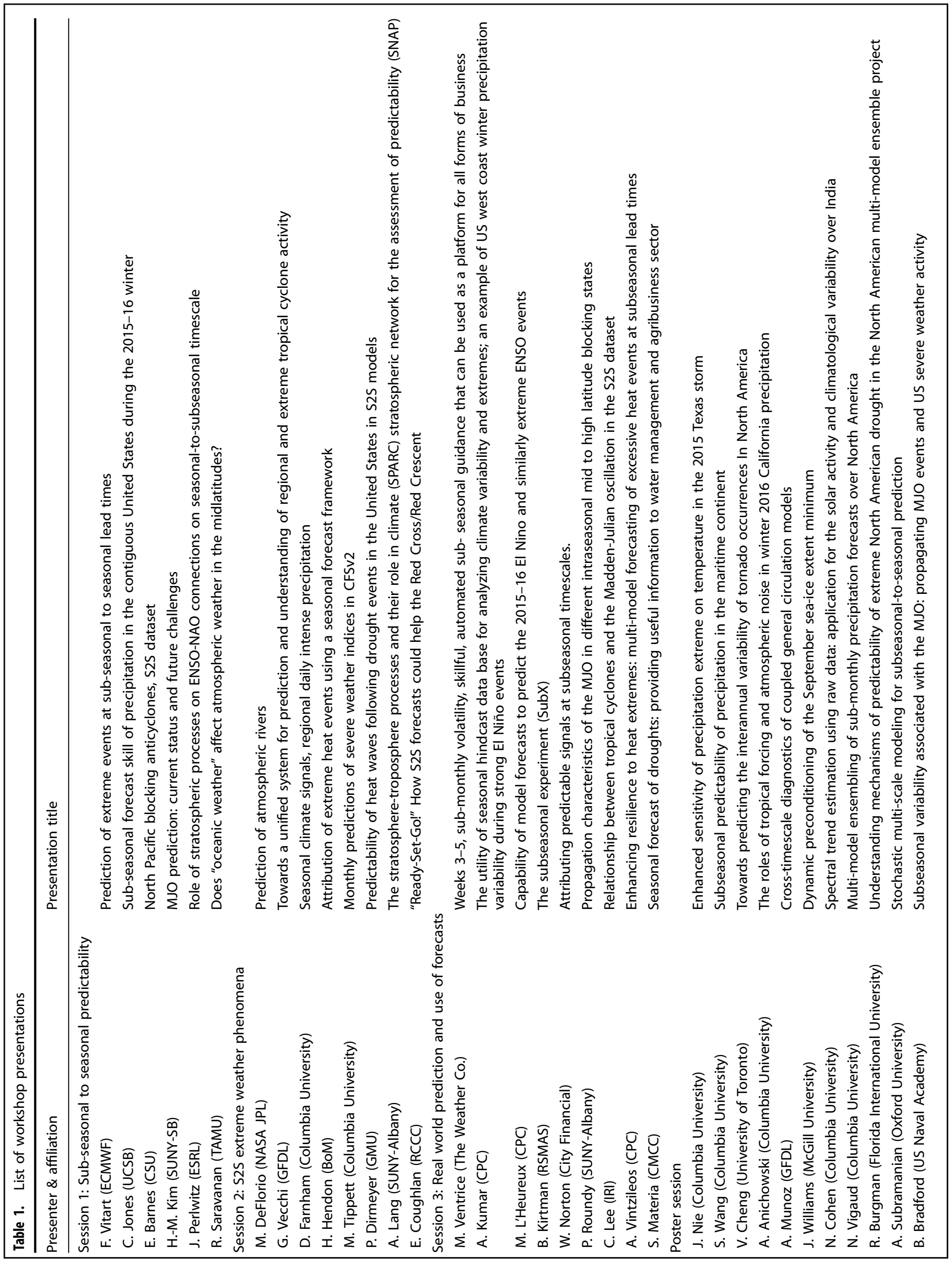




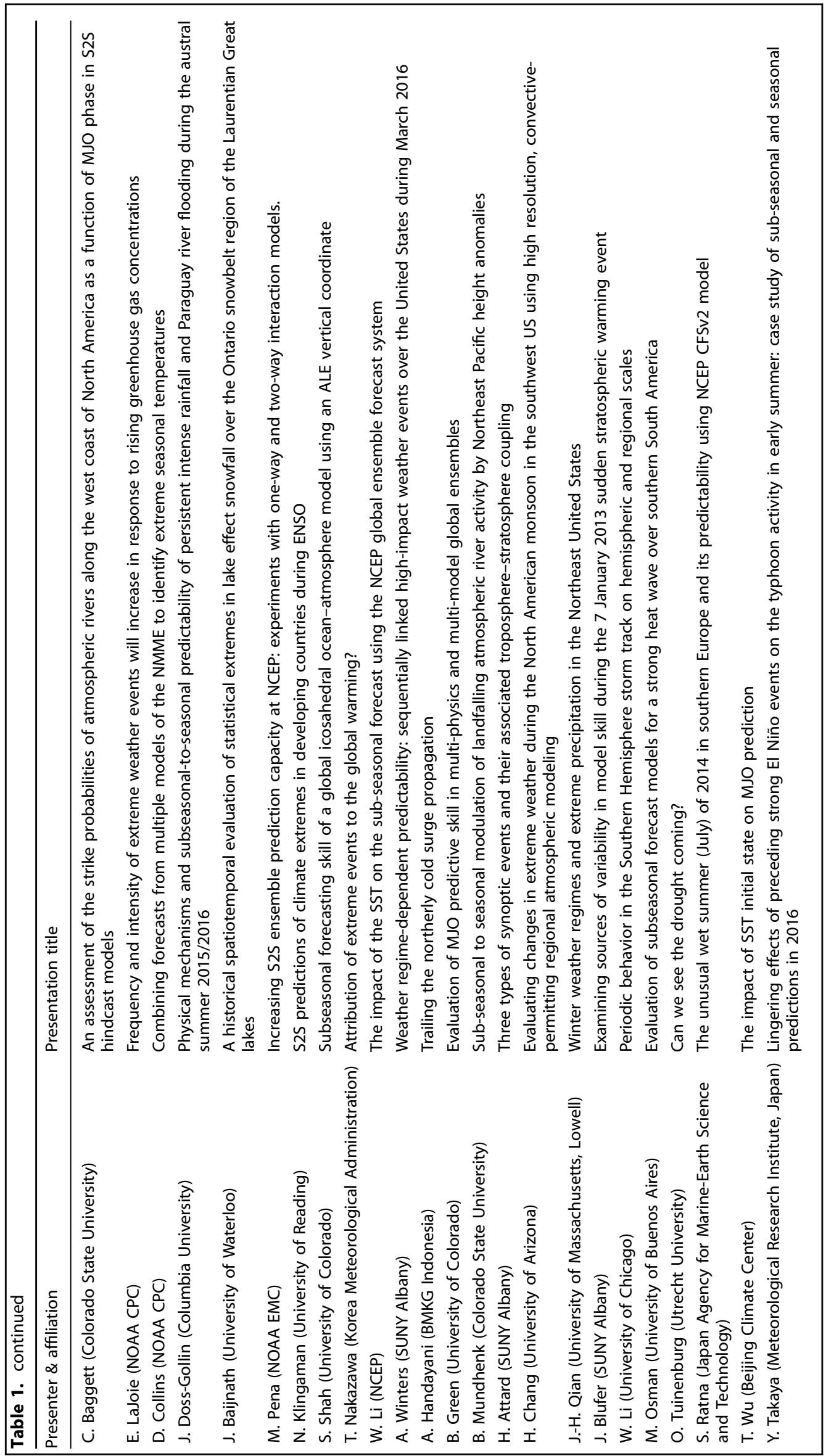


through the S2S database, and the workshop highlighted some of these developments. The database offers an unprecedented resource for scientists, modeling groups, and interested parties to better study, understand, and ultimately improve S2S prediction-in the same way that the Coupled Model Intercomparison Project (CMIP) archives have advanced climate change researchby making output from many models available publicly using a unified protocol. Several oral and poster presenters shared early results from the S2S database, which became fully operational in 2015, often highlighting the potential for future research. Several workshop presentations also featured the activities of the MAPP S2S Prediction Task Force, a research initiative to advance NOAA's capability to model and predict sources of S2S predictability. Specific S2S phenomena discussed included the MJO (H.-M. Kim, SUNY-SB), the role of stratospheric processes on ENSO-NAO teleconnections (J. Perlwitz, NOAA-ESRL), "oceanic weather" impacts on atmospheric midlatitude weather (R. Saravanan, TAMU), North Pacific blocking anticyclones (E. Barnes, CSU), and sub-seasonal forecast skill of precipitation in the contiguous United States during the 2015-16 winter (C. Jones, UCSB). A study of how MJO propagation in the tropics can be affected by midlatitude blocking was also presented ( $P$. Roundy, SUNYAlbany).

The MJO is recognized as one of the most important sources of predictability for sub-seasonal forecasts in the tropics, ${ }^{4}$ analogous to ENSO for seasonal forecasts, and a majority of the operational models now reach a bivariate anomaly correlation skill of the Wheeler-Hendon RMM indices exceeding 0.5 at 2 weeks lead time, quantified using the S2S database. ${ }^{5}$ This is remarkable advance over the situation even a decade ago when models were then not able to adequately represent and forecast the MJO. However, the MJO is often still too weak and too slowly propagating in models, and their forecasts still typically underestimate the forecast uncertainty. The impact of the MJO on weather conditions-particularly in the midlatitudes through teleconnections-is still not adequately captured by models, reducing the current value of S2S forecasts. Encouraging results are nonetheless starting to emerge, including predictability of the positive phase of the NAO up to 2-3 weeks in advance, which may be associated with MJO teleconnections.

The talk by $C$. Jones highlighted the challenges in forecasting precipitation beyond a week in advance over the US during the 2015-16 winter season. Blocking events over the North Pacific were shown by E. Barnes to steer Atmospheric Rivers into Alaska and the Pacific Northwest, which she was able to track to predictable MJO activity over the tropical Indio-Pacific Ocean, particularly during the easterly phase of the stratospheric quasi-biennial oscillation. The stratosphere is increasingly being recognized as an important player in forecasting on the S2S time scale and today's models often have a large number of vertical levels, allowing them to better resolve the stratosphere. The talk by J. Perlwitz drew attention to the role of sudden warmings of the polar stratosphere in mediating the impact of ENSO on the NAO. Midlatitude ocean eddies have monthly time scales and are garnering increasing attention as a potential source of the S2S predictability of US westcoast weather, as discussed by R. Saravanan.

\section{S2S EXTREME WEATHER PHENOMENA}

The second set of talks continued with the theme of predicting different types of extreme weather events, and how the latter are related to sub-seasonal predictable dynamical phenomena like the MJO. These included Atmospheric Rivers (M. DeFlorio, NASA JPL), tropical cyclones (G. Vecchi, GFDL; C. Lee IRI), intense precipitation and severe weather (D. Farnham, Columbia University; M. Tippett, Columbia University), heat waves and drought (H. Hendon, BoM; P. Dirmeyer, GMU). Tropical cyclone (TC) activity over Indo-Pacific Ocean is strongly modulated by the MJO, which provides a physical basis for sub-seasonal TC prediction. Encouraging analysis of forecasts from S2S database shows that the models can capture this modulation, and several speakers showed examples of successful TC activity forecasts, even from the lower-resolution models, while high-resolution models like the $25 \mathrm{~km}$ GFDL HiFLOR model are able to capture intense category 4 and 5 storms, which are not simulated by the lower resolution models. Although small-scale severe weather events, such as hail storms and tornados cannot be represented explicitly in the current generation of S2S forecast models, we may still be able to predict their aggregate statistics several weeks into the future. While the best skill is naturally found during the first week of the forecasts, M. Tippett (Columbia University) showed examples of index-based skillful prediction of tornado and hail activity over the US in the week 2-4 range using techniques developed for seasonal forecasting.

Several talks addressed the predictability of heat waves. Work over the US also highlighted the often strong relationship between preceding drought conditionals and heat waves ( $P$. Dirmeyer). Results from ECMWF indicate that the devastating Russian heat wave of 2010 could be have predicted up to 3 weeks in advance with today's models. The workshop also highlighted that S2S forecasts are now being used in extreme events attribution to physical phenomena. Results presented by F. Vitart showed that the extreme cold wave that occurred over Europe in March 2013 could partly be attributed to an MJO event over the Indian Ocean. The question of attributing the record breaking heat over Australia in October 2015 was addressed by $\mathrm{H}$. Hendon (BoM), who found that most of the predictability was supplied through atmospheric initial conditions up to 2 weeks in advance, with little role found for the ocean and land surface in that case. There is still much to be learned about the importance of atmospheric initial conditions near the classical deterministic predictability limit of Lorenz at 7-15 days.

\section{REAL WORLD PREDICTION AND USE OF FORECASTS}

A large part of the workshop was devoted to the emerging area of S2S forecasting for real-world applications. Besides promoting research to improve understanding and forecast skill, the WWRP/ WCRP's S2S project strives to spur forecast uptake by operational centers and exploitation by the applications community. The workshop witnessed considerable S2S interest in the private sector, with companies actively developing sub-seasonal forecasts and seeking to attribute skill to specific predictable phenomena, such as their MJO (M. Ventrice, The Weather Co.; W. Norton, City Financial). Such companies have previously used statistical models for energy forecasts and are eager to tap into new capabilities, including making weekly updates to established seasonal forecasts that are only issued once a month; the ability of sub-seasonal forecasts to provide weekly updates was particularly highlighted, as was the interest in temperature and wind forecasts.

Three presentations were made by members of NOAA's Climate Prediction Center (CPC), which has been issuing week $3+4$ probabilistic climate outlooks experimentally since September 2015 (A. Kumar, M. L'Heureux, A. Vintzileos). El Niño/La Niña events are important sources of predictability at sub-seasonal (multiweek), as well as seasonal (multi-month) forecast lead times, and the 2015-16 El Niño in particular was discussed. This event did not result in the expected wet conditions over California, and subseasonal forecasts provide a new tool for analyzing failures in seasonal forecasts by analyzing more frequently updated shorterterm forecast evolutions.

S2S forecast products are under development in several application sectors, and talks were presented on multi-model forecasting of excessive heat events (A. Vintzileos, CPC), lessons learned from seasonal forecast of droughts for the water management and agribusiness sectors (S. Materia, CMCC), and 
"Ready-Set-Go!", or how S2S forecasts could help the Red Cross/ Red Crescent (E. Coughlan, RCCC). Sub-seasonal forecasting of extremes will require more categories of the forecast distribution than the standard terciles used in seasonal forecasting, for instance, forecasts for extreme thresholds, such as the 85th or 90 th percentile, or the use of extremes indices. The choice of suitable metrics for verification of large numbers of quantiles were discussed, as well as the difficulty of identifying suitable "danger level" thresholds, for which the "cost-loss" model framework may be informative.

For real-world forecast applications, it will be necessary to have access to S2S forecasts in real time. While the S2S database is purely for research purposes and only provides these forecasts with a 3-week delay, at least two initiatives will soon provide the forecasts in real time. The WMO is working through its Lead Centre for long-range forecasting to create multi-model products in real time from the S2S models that will be made accessible to national meteorological services and the WMO's regional climate centres. The second initiative is the NOAA program new Subseasonal Experiment (SubX; http://cola.gmu.edu/kpegion/subx) (presented by B. Kirtman, RSMAS) that will provide sub-seasonal real-time forecasts and hindcasts from North American modeling centers (NCEP, Environment Canada, US Navy, NASA, NOAA-ESRL, and NCAR/COLA/RSMAS) in a publicly accessible database housed at IRI-Columbia University. SubX predictions will not only be realtime but will also adhere to a common forecasting protocol, which will facilitate both research and real-world applications, extending what is possible based on the S2S database.

\section{CLOSING REMARKS}

The S2S time scale is a frontier in our efforts to gain understanding and improve prediction of weather and climate, and progress will naturally require collaboration among individuals from diverse communities and viewpoints. S2S research is an emerging area where there are ample opportunities for young researchers to contribute to advancing scientific understanding, as well as the possibility to have meaningful impacts on the business, government, and nonprofit sectors.

\section{ACKNOWLEDGEMENTS}

We wish to thank all the presenters and participants at the workshop, and give special thanks to Jaclyn Rabinowitz and Dannie Dinh whose excellent local organization and logistics enabled a successful workshop. It is a pleasure to thank two anonymous reviewers whose comments improved the quality of the manuscript. The organizers of the workshop are grateful for the support provided by Columbia University's Extreme Weather \& Climate Initiative, WMO, the NOAA Climate Program Office Modeling,
Analysis, Predictions, and Projections (CPO-MAPP) program, and Columbia University's International Research Institute for Climate and Society (IRI) which co-organized and hosted the event in its Monell Auditorium in Palisades, New York.

\section{AUTHOR CONTRIBUTIONS}

All authors researched, collated, and wrote this paper.

\section{ADDITIONAL INFORMATION}

Competing interests: The authors declare no competing financial interests.

Publisher's note: Springer Nature remains neutral with regard to jurisdictional claims in published maps and institutional affiliations.

Change history: The original version of this Article had an incorrect Article number of 8 and an incorrect Publication year of 2017. These errors have now been corrected in the PDF and HTML versions of the Article.

\section{REFERENCES}

1. Vitart, F., Robertson, A. W. and S2S Steering Group. Sub-seasonal to seasonal prediction: linking weather and climate. In Seamless Prediction of the Earth System: From Minutes to Months, WMO-1156 (eds Brunet, G., Jones, S. \& Ruti, P. M.) 385-401 (World Meteorological Organization, Geneva, Switzerland, 2015).

2. Vitart, F., Robertson, A. W. \& Anderson, D. L. T. Subseasonal to seasonal prediction project, 2012: bridging the gap between weather and climate. WMO Bull. 61(2), 23-28 (2012).

3. Vitart, F. et al. The Subseasonal to Seasonal (S2S) prediction project database. Bull. Am. Meteor. Soc. 98(1), 163-176 (2017).

4. Zhang, C. Madden-Julian Oscillation: bridging weather and climate. Bull. Am. Meteor. Soc. 94, 1849-1870 (2013).

5. Vitart, F. Madden-Julian Oscillation prediction and teleconnections in the S2S database: MJO prediction and teleconnections in the S2S database. Q. J. R. Meteor. Soc. 143, 2210-2220 (2017).

\footnotetext{
(c) (i)

Open Access This article is licensed under a Creative Commons Attribution 4.0 International License, which permits use, sharing, adaptation, distribution and reproduction in any medium or format, as long as you give appropriate credit to the original author(s) and the source, provide a link to the Creative Commons license, and indicate if changes were made. The images or other third party material in this article are included in the article's Creative Commons license, unless indicated otherwise in a credit line to the material. If material is not included in the article's Creative Commons license and your intended use is not permitted by statutory regulation or exceeds the permitted use, you will need to obtain permission directly from the copyright holder. To view a copy of this license, visit http://creativecommons org/licenses/by/4.0/.
}

(c) The Author(s) 2018 